\title{
3 Research Square

\section{Title Anterior Prominence of the Femoral Condyle Varies among Prosthesis Designs and Surgical Techniques in Total Knee Arthroplasty}

Junya Itou

Tokyo Women's Medical University

Umito Kuwashima

Tokyo Women's Medical University

Masafumi Itoh

Tokyo Women's Medical University

Ken Okazaki ( $\Delta$ okazaki.ken@twmu.ac.jp )

Tokyo Women's Medical University

\section{Research Article}

Keywords: Total knee arthroplasty, Computer simulation, Patellofemoral overstuffing, Anterior reference, Posterior reference

Posted Date: July 21st, 2021

DOl: https://doi.org/10.21203/rs.3.rs-731593/v1

License: (9) This work is licensed under a Creative Commons Attribution 4.0 International License. Read Full License 


\section{Abstract}

\section{Background}

Patellofemoral overstuffing after total knee arthroplasty can cause limited range of motion and anterior knee pain. This study compared anterior prominence of femoral components among different prothesis designs in surgical simulation models utilizing the anterior reference (AR) and posterior reference (PR) techniques.

\section{Methods}

Surgical simulations were performed using preoperative computed tomography data of 30 patients on a three-dimensional planning system. Four implant models were used: Attune, Persona, Journey II, and Legion. Rotational alignment was set parallel to the transepicondylar axis and size selection was based on absence of notch formation in the femoral anterior cortex and best fit to the shape of the medial posterior femoral condyle. For each combination of surgical technique (AR or PR method) and implant model, measurements were taken of the maximum medial, central, and lateral prominence of the implant from the anterior femoral cortex $(\mathrm{mm})$.

Results

Using either the AR or PR method, the medial and central prominences were significantly lower with Journey II than with other models. The lateral prominence was the lowest with Attune in the AR method. The AR method was associated with significantly less prominence than the PR method, regardless of implant model.

\section{Conclusions}

Degree of anterior prominence of the femoral implant is affected by implant design when the AR method is used. The PR method is associated with greater anterior prominence compared with the AR method, with size pitch an additional influencing factor. Journey II is associated with the least anterior prominence when using either method.

\section{Introduction}

Total knee arthroplasty (TKA) is widely known to provide good postoperative outcomes, with welldocumented effectiveness in relieving pain and achieving good range of motion (ROM) $[1,2,3]$. Postoperative ROM is one of the factors associated with postoperative physical function [4] and patient satisfaction [5]. Various factors have been reported to influence postoperative ROM, including ligament balance, preoperative ROM, posterior condylar offset, and low anterior femoral condylar height [6, 7]. Recently, it has been reported that reducing the size of the anterior condylar height after TKA can improve flexion [8]. Anterior prominence of the femoral component has been suggested to influence postoperative 
ROM because it can cause overstuffing of the patellofemoral (PF) joint. This overstuffing is known to affect postoperative ROM [9] and can cause anterior knee pain [10].

Various factors have been associated with PF overstuffing, such as surgical technique and component design and size. Component resizing has been associated with increased PF pressure during knee flexion in the swing phase of gait [11]. Differential model designs have been associated with differential PF pressure and patellar kinematics [12]. Even when adopting the same surgical technique, implant selection can result in different degrees of anterior femoral prominence because of both the design and pitch size of implants. However, this issue has not been focused on, and no information is available about the difference in anterior prominence of the femoral component among implant designs.

The objective of this study was to investigate differences in anterior prominence of the femoral components among different models when the prosthesis was implanted in the same patients with the same surgical techniques. To achieve this, we adopted a three-dimensional (3D) surgical simulation model to measure the anterior prominence of femoral condyle of several widely used prostheses placed with the anterior reference or posterior reference method.

We hypothesized that anterior prominence is minimized with implant models that have a thin anterior flange when using the anterior reference method or models that have a generous pitch size when using the posterior reference method.

The clinical relevance of this study is that the findings obtained would remind surgeons of the need to pay more attention to pitch size and design of the anterior flange in choosing or developing a new implant.

\section{Materials And Methods}

A total of 30 patients (12 men, 18 women; mean age 72.7 years, age range $53-85$ years) with knee osteoarthritis (OA) scheduled to undergo TKA were recruited into the study. In 11 patients, knees were classified as Kellgren-Lawrence Grade 3 and in 19 patients as Grade 4. Twenty-six patients had varus OA with a mean hip-knee-ankle angle of $170.9 \pm 5.9^{\circ}\left(156.0-179.0^{\circ}\right)$, and 4 had valgus $O A$ with a mean hipknee-ankle angle of $185.2 \pm 1.2^{\circ}\left(184.2-186.6^{\circ}\right)$ (Table 1$)$. Surgical simulation was performed using preoperative computed tomography (CT) data on a 3D planning system (Zed Knee; LEXI, Tokyo, Japan). A femoral implant was placed perpendicular to the 3D mechanical axis for coronal and sagittal alignment. Rotational alignment was set parallel to the surgical transepicondylar axis (SEA). Using the anterior reference method, the implant was placed so that the posterior of the anterior flange was in contact with the anterior cortex, and size was determined based on the best fit to the medial posterior condyle. Using the posterior reference method, the implant was placed so that it was in contact with the posterior border of the medial posterior condyle, and the smallest size that did not form a notch in the anterior cortex was selected (Fig. 1). Four implant models were used: Attune PS (DePuy, Warsaw, IN; "Attune"); Persona PS (Zimmer-Biomet, Warsaw, IN; "Persona”); Journey II BCS (Smith \& Nephew, Memphis, TN; “Journey II”); and Legion PS (Smith \& Nephew; "Legion”). For each combination of surgical 
technique and implant model, the maximum medial, central, and lateral prominence of the implant from the anterior femoral cortex ( $\mathrm{mm}$ ) and component size were measured (Fig. 2).

Table 1

Demographic data of 30 patients with knee osteoarthritis scheduled to undergo total knee arthroplasty

\begin{tabular}{|ll|}
\hline Age (years) & $\mathbf{7 2 . 7} \pm 6.9$ (53-85) \\
\hline Sex (M / F) & $12 / 18$ \\
\hline $\mathrm{BMI}\left(\mathrm{kg} / \mathrm{m}^{2)}\right.$ & $26.6 \pm 4.6(18.0$ to 36.5$)$ \\
\hline Affected side (Rt / Lt) & $15 / 15$ \\
\hline
\end{tabular}

\subsection{Statistical analysis}

The Steel-Dwass test was used for multiple comparisons between the four implant groups; Wilcoxon's signed-rank test was used for paired comparison of the anterior reference versus posterior reference method with the same implants. Statistical significance was based on $p$-values of less than $5 \%$. For the measurement of intra-observer reliability, the maximum medial, central, and lateral prominences were measured twice at an interval of $\geq 14$ days for each implant model in 4 randomly selected patients. The resulting intraclass correlation coefficient $($ ICC 1,1$)$ was 0.94 . In statistical power analysis, assuming a power of 0.8 , alpha of 0.05 , and standard deviation of 0.5 , the sample size to detect a difference in prominence between models of $0.5 \mathrm{~mm}, 0.5 \mathrm{~mm}$, and $1 \mathrm{~mm}$, respectively, was calculated as 27 .

\subsection{Ethical approval and consent to participate}

All procedures involving human participants were in accordance with the ethical standards of the 1964 Helsinki Declaration and its later amendments. This study was conducted with approval from the ethics committees of Tokyo Women's Medical University (approval no.4578, December 12, 2017). Informed consent was obtained via an opt-out procedure.

\section{Results}

The anatomical parameters used for implant placement in the surgical simulation for 30 patients are listed in Table 2. Mean valgus angle against the distal anatomical axis of the femur was $6.3 \pm 1.9$ and mean external rotation angle from the posterior condylar axis was $3.8 \pm 1.6^{\circ}$. The mean sagittal plane angle against the distal bone axis was $1.3 \pm 1.9^{\circ}$ extension. 
Table 2

Anatomical parameters

Valgus ( $\left(^{\circ}\right)$

External rotation $\left(^{\circ}\right)$

Sagittal alignment**( $\left(^{\circ}\right)$

*Expressed as an external rotation angle from the posterior condylar angle

${ }^{*}$ Negative values indicate extension and positive values indicate flexion against the distal bone axis. Journey II than with the other models $(p<0.001$, Fig. 3$)$. The lateral prominence was significantly lower with Attune than with the other models, with a $0.2-\mathrm{mm}$ difference compared with Journey II $(p<0.02$, Fig. $3)$.

With the posterior reference method, the medial and central prominences were significantly lower with Journey II than with the other models ( $p<0.0001$, Fig. 4). Lateral prominence was significantly lower with Attune, Journey II, and Persona than with Legion ( $p<0.0001$, Fig. 4), with no significant difference among the Attune, Journey II, and Persona models ( $p>0.21$, Fig. 4).

With the posterior reference method, component upsizing was needed to avoid anterior notch formation in $4,3,5$, and 8 cases placed with Attune, Persona, Journey II, and Legion, respectively.

For each implant model, the medial, central, and lateral prominences were significantly lower using the anterior reference method than the posterior reference method $(p<0.05$, Fig. 5$)$.

\section{Discussion}

The most important finding of this study was that the anterior prominence of the femoral condyle varied significantly depending on the implant design in either the anterior reference or posterior reference method. Journey II provided the least medial and central prominence among the 4 designs investigated. The anterior flange of Journey II is thinner than that of other models, suggesting the design influences the results significantly. In the posterior reference method, Attune and Persona were also associated with lower anterior prominence, probably because the generous pitch sizes of these models allowed for the selection of the size most closely attached to the anterior cortex.

Anterior prominence of the femoral component can lead to PF overstuffing and can affect ROM [9]. Nishitani et al. [8] have reported that a decrease in the height of the anterior condyle from the original level is associated with increased postoperative flexion. Relatively good clinical outcomes in terms of postoperative ROM have been reported with Journey II. Brilhault et al. reported a mean ROM of $124 \pm 9.8^{\circ}$ [1]. Vascellari et al. described improved mean ROM from $99.3^{\circ}$ preoperatively to $119^{\circ}$ postoperatively [13], and Taniguchi et al. reported a corresponding improvement from $117.7^{\circ}$ (SD15.3 ${ }^{\circ}$ ) to $129.0^{\circ}$ (SD $\left.9.6^{\circ}\right)$ [14]. Although these reports did not compare Journey II with other models under the same conditions, the 
relatively good ROM results reported with Journey II may be partially attributable to lower anterior femoral prominence than with other models. Selecting an implant with a thin flange may be a good strategy for gaining better postoperative ROM. In the anterior reference method, the degree of anterior prominence of the implant is likely affected by the implant design.

In contrast, using the posterior reference method, other factors are suspected to be involved. When an implant has been selected based on the best fit to the shape of the medial condyle, upsizing would be needed if an anterior notch is formed. In such cases, the use of models with generous pitch sizes is likely to result in a smaller mean anterior prominence. In fact, relatively small numbers of patients treated with Persona or Attune required implant upsizing, likely because of the generous pitch size and design of these models. Persona has a pitch size of $2 \mathrm{~mm}$ and Journey II and Attune have a pitch size of $3 \mathrm{~mm}$. In contrast, Legion was associated with higher anterior prominence, likely due to its design and pitch size. More specifically, its pitch size of $4 \mathrm{~mm}$ and greater flange thickness compared with the other models appeared greatly impact on the outcome.

The decision to select the anterior reference or posterior reference method in an actual operation depends on the mechanical property of the implant and the surgical technique to be used. It is generally understood that when using the anterior reference method, notch formation can be avoided, but it is associated with difficulty in adjusting the posterior condylar offset and flexion gap. In contrast, the posterior reference method is associated with easier adjustment of the posterior condylar offset and flexion gap, but with higher risks of notch formation and PF overstuffing [15]. The present study did not take into account compatibility to the size of tibial implant component or the flexion gap. Therefore, in actual surgery, surgeons should be cautious in selecting surgical techniques and procedures.

Nevertheless, the fact that Journey II provided less anterior prominence in either the anterior reference or posterior reference method suggests that the implant design significantly affects it whichever method is to be used.

In this study, we defined implant placement on the coronal and sagittal planes perpendicular to the 3D functional axis. The mean valgus angle was $6.3^{\circ}$, which is comparable to that reported previously in a study using 3D-CT $\left(5.4 \pm 0.7^{\circ}\right)[16]$ and in another study $\left(6.3^{\circ}\right.$ when limited to knees with severe genu varum) [17]. The transepicondylar axis (TEA) can be either the clinical epicondylar axis or SEA. In this study, the SEA was used based on previous CT-based studies $[18,19]$. Meric et al. [20] defined the SEA as externally rotated by a mean of $3.3 \pm 1.5^{\circ}$ from the posterior condylar axis in a CT analysis of 13,546 knees. Victor [21] has concluded that the SEA is externally rotated by a mean of $3^{\circ}$ from the posterior condylar axis based on a review of rotation data. These values are consistent with corresponding values obtained from the patients analyzed in this study. Mean implant angle on the sagittal plane against the distal femoral axis was $1.3 \pm 1.9^{\circ}$ extension, which was considered to be prone to notch formation. However, this was unlikely to affect the results of the present study because differences between implants were investigated using surgical simulation under the same condition of placing the implant perpendicular to the 3D functional axis. 
There are several limitations to this study. First, we evaluated only 4 implant models. In this study, we sought to learn how implant design and sizing affect the anterior prominence rather than to search for the best implant available, and we selected models that are representative of the most commonly used models from each manufacturer in the registry. It is recommended that surgeons pay more attention to the design of anterior flange of the implant they use. Second, the femoral external rotation angle was only set against the SEA. We also evaluated all cases by setting the external rotation angle as being externally rotated by $3^{\circ}$ from the posterior condylar line and obtained similar results. Therefore, only the data aligned to the SEA was presented to avoid confusion.

\section{Conclusions}

The degree of anterior prominence of the femoral implant is affected by implant design when the anterior reference method is used. The posterior reference method is associated with greater anterior prominence compared with the anterior reference method, with pitch size identified as an additional influencing factor. Journey II is associated with the least anterior prominence when using either method. The various implant designs were shown to be associated with different degrees of anterior prominence, so additional attention is needed to the effects of surgical techniques and pitch size. In terms of relevance to daily clinical practice, our findings highlight the need for surgeons to be familiar with implant designs, including the thickness of the anterior flange and available size selections, to ensure favorable postoperative outcomes.

\section{Abbreviations}

$\mathrm{CT}$, computed tomography; TKA, total knee arthroplasty; PF, patellofemoral; ROM, range of motion

\section{Declarations}

\section{Ethics approval and consent to participate}

All procedures involving human participants were in accordance with the ethical standards of the 1964 Helsinki Declaration and its later amendments. This study was conducted with approval from the ethics committees of Tokyo Women's Medical University (approval no.4578, December 12, 2017). Informed consent was obtained via an opt-out procedure.

\section{Consent for publication}

Not applicable.

\section{Availability of data and materials}


The datasets used and/or analysed during the current study are available from the corresponding author on reasonable request.

\section{Competing interests}

K.O. received speaker's fee for Smith and Nephew, Inc. and Zimmer-Biomet. The other authors declare that they have no competing interests.

\section{Funding}

No funding was received for this work.

\section{Author contributions}

JI: Data collection, data analysis, writing the manuscript

UK: Revised the manuscript

Ml: Revised the manuscript

KO: Study design, data analysis, revised the manuscript

All authors read and approved the final manuscript

\section{Acknowledgements}

We thank ThinkSCIENCE for English language editing.

\section{References}

1. Brilhault J, Ries MD. Measuring patellar height using the lateral active flexion radiograph: effect of total knee implant design. Knee 2010;17:148-151.

2. Ethgen O, Bruyere O, Richy F, Dardennes C, Reginster JY. Health-related quality of life in total hip and total knee arthroplasty. A qualitative and systematic review of the literature. J Bone Joint Surg Am 2004;86:963-974.

3. Kane RL, Saleh KJ, Wilt TJ, Bershadsky B. The functional outcomes of total knee arthroplasty. J Bone Joint Surg Am 2005;87:1719-1724.

4. Devers BN, Conditt MA, Jamieson ML, Driscoll MD, Noble PC, Parsley BS. Does greater knee flexion increase patient function and satisfaction after total knee arthroplasty? J Arthroplasty 2011;26:178186. 
5. Matsuda S, Kawahara S, Okazaki K, Tashiro Y, Iwamoto Y. Postoperative alignment and ROM affect patient satisfaction after TKA. Clin Orthop Relat Res 2013;471:127-133.

6. Kim YH, Sohn KS, Kim JS. Range of motion of standard and high-flexion posterior stabilized total knee prostheses. A prospective, randomized study. J Bone Joint Surg Am 2005;87:1470-1475.

7. Stryker LS, Odum SM, Springer BD, Fehring TK. Role of patellofemoral offset in total knee arthroplasty: a randomized trial. Orthop Clin North Am 2017;48:1-7.

8. Nishitani K, Hatada R, Kuriyama S, et al. A greater reduction in the distal femoral anterior condyle improves flexion after total knee arthroplasty in patients with osteoarthritis. Knee 2019;26(6):13641371.

9. Mihalko W, Fishkin Z, Krackow K. Patellofemoral overstuff and its relationship to flexion after total knee arthroplasty. Clin Orthop Relat Res 2006;449:283-287.

10. Pierson JL, Ritter MA, Keating EM, et al. The effect of stuffing the patellofemoral compartment on the outcome of total knee arthroplasty. J Bone Joint Surg Am 2007;89:2195-2203.

11. Kawahara S, Matsuda S, Fukagawa S, et al. Upsizing the femoral component increases patellofemoral contact force in total knee replacement. J Bone Joint Surg Br 2012;94:56-61.

12. Tanikawa H, Tada M, Harato K, Okuma K, Nagura T. Influence of total knee arthroplasty on patellar kinematics and patellofemoral pressure. J Arthroplasty 2017;32:280-285.

13. Vascellari A, Schiavetti S, Rebuzzi E, Coletti N. Functional versus patient-reported outcome of the bicruciate and the standard condylar-stabilizing total knee arthroplasty. Eur J Orthop Surg Traumatol 2016;26:305-310.

14. Taniguchi $\mathrm{H}$, Itoh M, Yoshimoto N, Itou J, Kuwashima U, Okazaki K. Noise after total knee arthroplasty has limited effect on joint awareness and patient-reported clinical outcomes: retrospective study. BMC Musculoskelet Disord 2020;21:115.

15. Charette RS, Sheth NP, Boettner F, Scuderi GR, Melnic CM. Femoral component sizing during total knee arthroplasty: anterior versus posterior referencing. JBJS Rev 2018;6(1):e4. https://doi.org/10.2106/JBJS.RVW.17.00051

16. Jamali AA, Meehan JP, Moroski NM, Anderson MJ, Lamba R, Parise C. Do small changes in rotation affect measurements of lower extremity limb alignment? J Orthop Surg Res 2017;12:77-84.

17. Sailhan F, Jacob L, Hamadouche M. Differences in limb alignment and femoral mechanicalanatomical angles using two dimension versus three dimension radiographic imaging. Int Orthop 2017;41:2009-2016.

18. Kobayashi H, Akamatsu Y, Kumagai K, Kusayama Y, Aratake M, Saito T. Is the surgical epicondylar axis the center of rotation in the osteoarthritic knee? J Arthroplasty 2015;30:479-483.

19. Oshima Y, lizawa N, Kataoka T, Majima T, Takai S. A computed-tomography-scan-based template to place the femoral component in accurate rotation with respect to the surgical epicondylar axis in total knee arthroplasty. Knee 2018;25:195-202. 
20. Meric G, Gracitelli GC, Aram LJ, Swank ML, Bugbee WD. Variability in distal femoral anatomy in patients undergoing total knee arthroplasty: measurements on 13,546 computed tomography scans. J Arthroplasty 2015;30:1835-1838.

21. Victor J. Rotational alignment of the distal femur: a literature review. Orthop Traumatol Surg Res 2009;95:365-372.

\section{Figures}

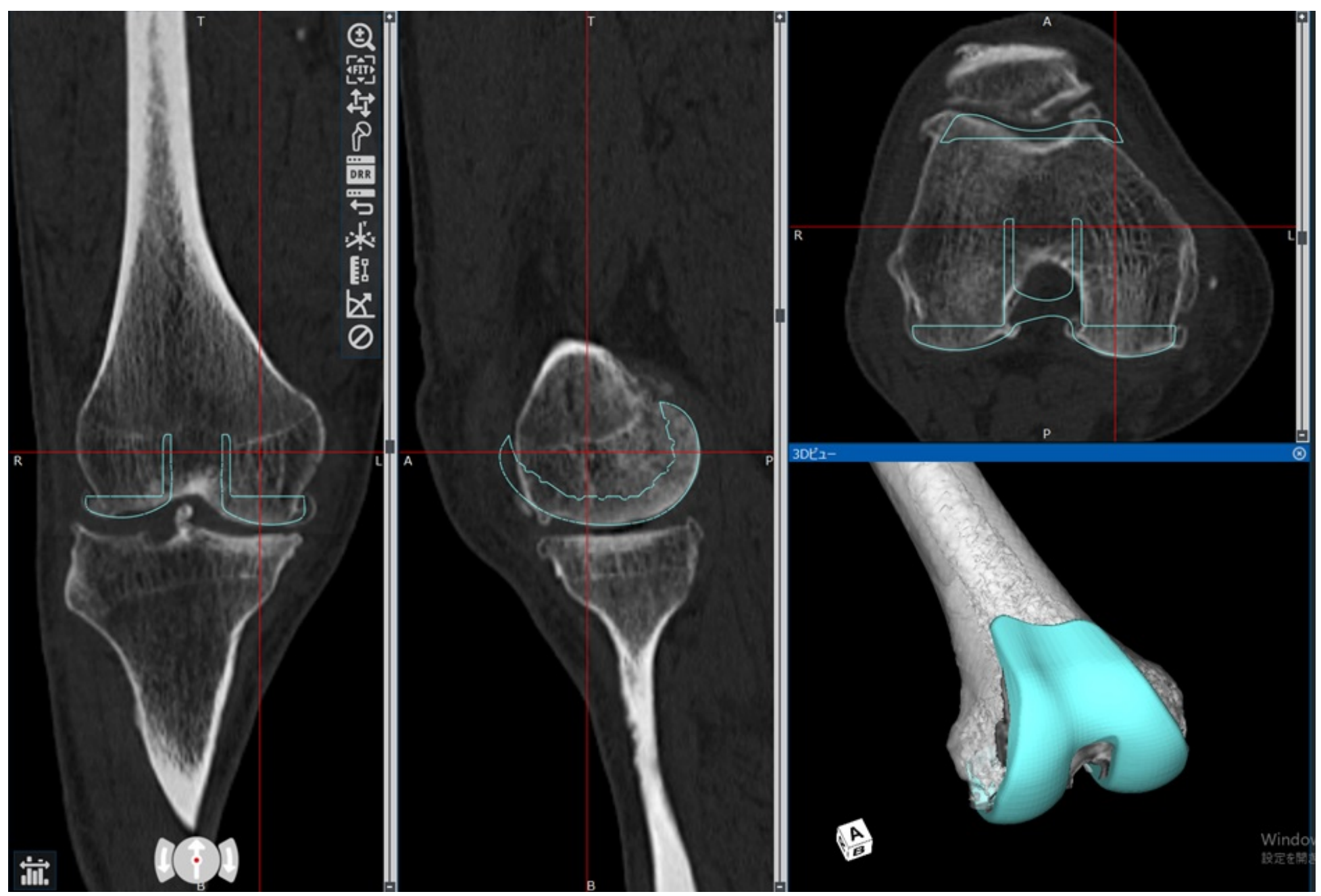

\section{Figure 1}

Representative implant placement simulation (Journey II, right knee osteoarthritis) Femoral implant is placed perpendicular to the three-dimensional functional axis for varus/valgus deformity and extension/flexion. Rotational alignment is set parallel to the transepicondylar axis (surgical epicondylar axis). Using the anterior reference method, the implant is placed so that the posterior of the anterior flange is in contact with the anterior cortex, and size selection is based on the best fit of the medial posterior condyle of the implant. With the posterior reference method, the implant is placed in contact with the posterior border of the medial posterior condyle, and the smallest size that did not form a notch in the anterior cortex is selected. 
(1)

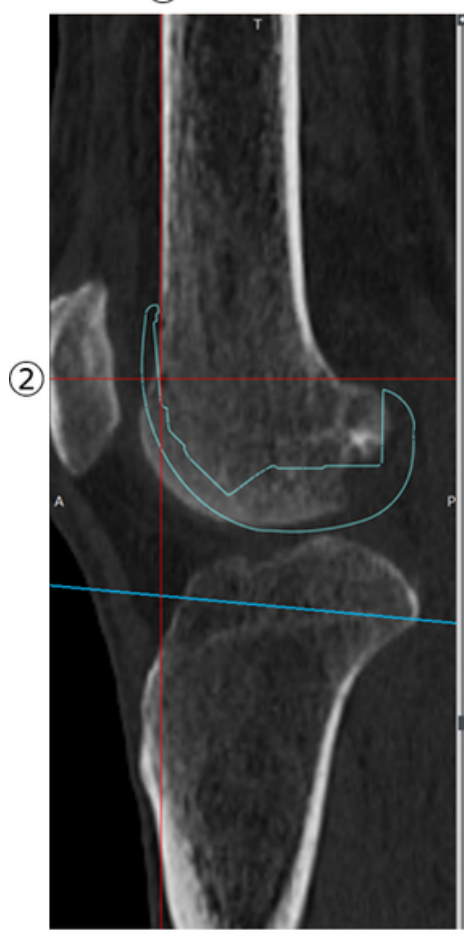

(2)

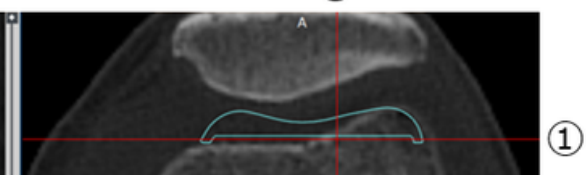

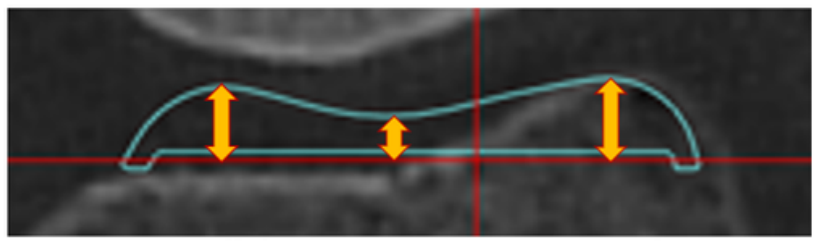

Medial Center Lateral

Bir

\section{Figure 2}

Details of measuring the anterior prominence Measurements of the maximum medial, central, and lateral prominences of the implant from the anterior femoral cortex are shown (red line 1; double arrows).
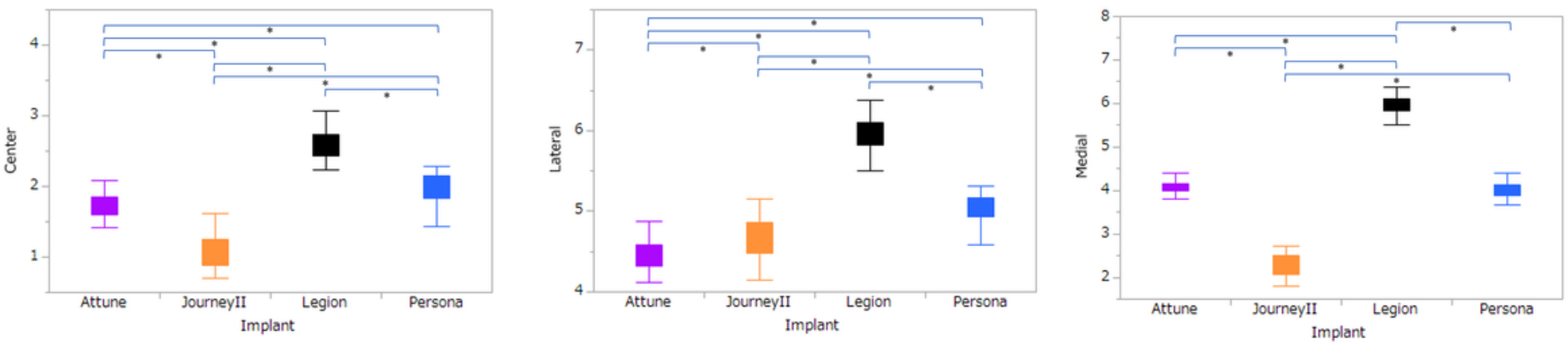

\section{Figure 3}

Comparison of medial, central, and lateral prominences $(\mathrm{mm})$ using the anterior reference method with different implants *Steel-Dwass test With the anterior reference method, the medial and central prominences were significantly lower with Journey II than with the other models $(p<0.001)$. The lateral prominence was significantly lower with Attune than with the other models $(p<0.02)$. 

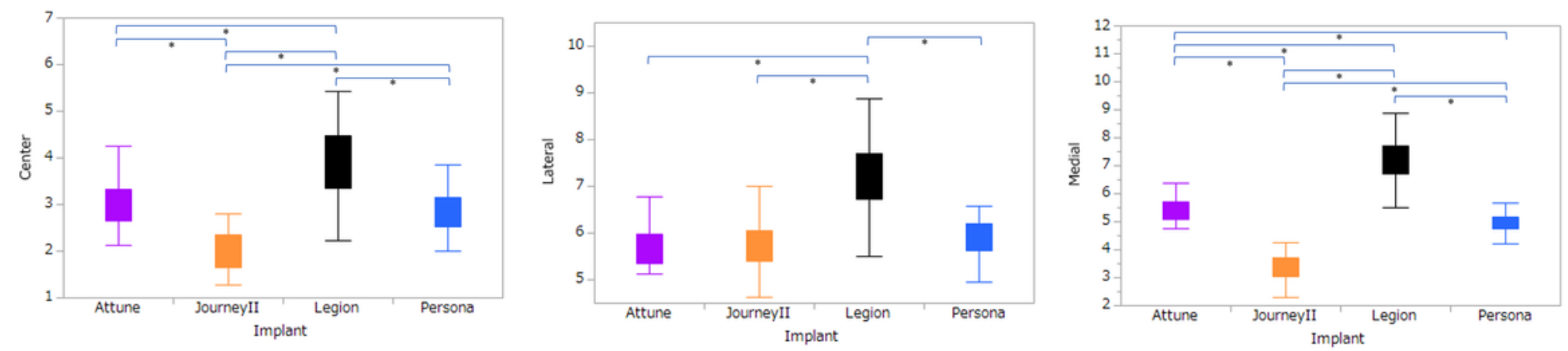

Figure 4

Comparison of medial, central, and lateral prominences $(\mathrm{mm})$ using the posterior reference method with different implants * Steel-Dwass test With the posterior reference method, the medial and central prominences were significantly lower with Journey II than with the other models $(p<0.0001)$. The lateral prominence was significantly lower with Attune, Journey II and Persona than with Legion $(p<0.0001)$, with no significant difference among the Attune, Journey II, and Persona models ( $p>0.21)$.
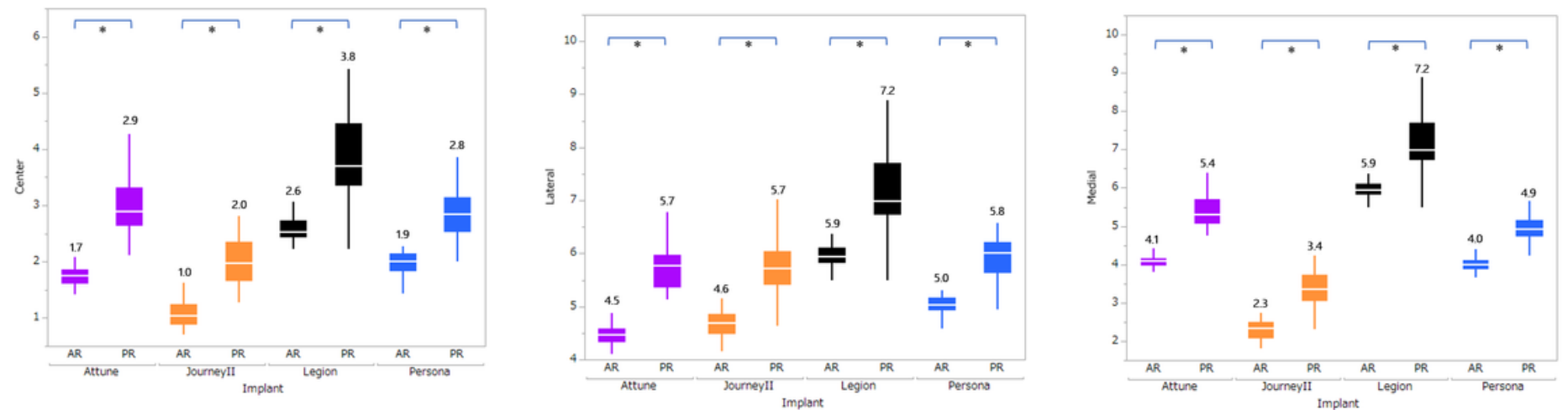

Figure 5

Comparison of anterior and posterior reference methods *Wilcoxon's signed-rank test For all implants, the anterior reference method was associated with significantly lower anterior prominence $(p<0.05)$. 